\title{
Effect of a microtubule-targeting drug on cell-cell contacts in bladder epithelial tumour cells
}

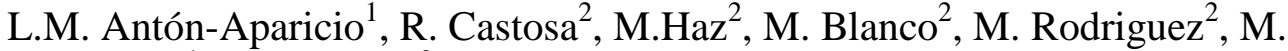 \\ Valladares $^{1}$, A. Figueroa ${ }^{2}$. \\ ${ }^{1}$ Complejo Hospitalario Universitario A Coruña (INBIC-CHUAC), Medical Oncology Unit, Coruña, Spain; ${ }^{2}$ \\ Instituto Investigación Biomédica A Coruña-Complejo Hospitalario Universitario A Coruña (INBIC-CHUAC), \\ Translational Cancer Research Group, Coruña, Spain
}

Background: Bladder cancer is a common malignancy affecting the genitourinary system that represents the fifth most common cancer in the world. Transitional cell carcinoma (TCC) represents $95 \%$ of these tumours. Vinflunine (VFL) is a microtubule-targeting drug that suppresses microtubule dynamics, showing anti-metastatic properties both in vitro and in living cancer cells. An increasing body of evidence underlines the influence of the microtubules dynamics on the cadherin-dependent cell-cell adhesions. In this report, we investigate the role of VFL on cell-cell contacts in bladder epithelial tumour cells.

Methods: Human bladder epithelial tumour cell lines HT1376, 5637 and UMUC3 were used to analyse cadherin-dependent cell-cell adhesions under VFL treatment. VFL effect on growth inhibition was measured by using a MTT colorimetric cell viability assay. Western blot, immunofluorescence and transmission electron microscopy analyses were performed to assess the roles of VFL effect on cell-cell adhesions, epithelial-to-mesenchymal markers and apoptosis. The role of the proteasome in controlling cell-cell adhesion was studied using the proteasome inhibitor MG132.

Results: We show that VFL induces cell death in bladder cancer cells and activates epithelial differentiation of the remaining living cells, leading to an increase of E-cadherin-dependent cell-cell adhesion and a reduction of mesenchymal markers, such as $\mathrm{N}$-cadherin or vimentin. Moreover, while Ecadherin is increased, the levels of Hakai, an E3 ubiquitin-ligase for E-cadherin, were significantly reduced in presence of VFL. In 5637, this reduction on Hakai expression was blocked by MG132 proteasome inhibitor, indicating that the proteasome pathway could be one of the molecular mechanisms involved in its degradation.

Conclusions: Our findings underscore a critical function for VFL in cell-cell adhesions of epithelial bladder tumour cells, suggesting a novel molecular mechanism by which VFL may impact upon EMT and metastasis. 